\title{
Transient nonlinear optical behavior of fluorescein-doped boric acid glass
}

\author{
Colette Maloney* and Werner Blau \\ Department of Pure and Applied Physics, Trinity College, Dublin 2, Ireland
}

\begin{abstract}
Received October 10, 1990; revised manuscript received February 12, 1992
The nonlinear optical properties of fluorescein-doped boric acid glass films have been investigated with subnanosecond laser pulses. With the use of degenerate four-wave mixing, large third-order susceptibilities of $\chi^{(3)} \sim 10^{-7}$ esu have been measured, connected to the relatively fast decay of the singlet state $S_{1}$ $(\tau=2.4 \pm 0.6 \mathrm{~ns})$. This particular system has a long-lived lowest-lying triplet state $(0.1-1 \mathrm{~s})$, and the transient nonlinear-optical and fluorescence properties show a marked dependence on the laser-pulse repetition rate in this time regime.
\end{abstract}

\section{INTRODUCTION}

Recent nonlinear-optical studies of dyes in solid hosts have been prompted by the low saturation intensities of such materials, which lead to relatively high nonlinearities stemming from nonlinear absorption. ${ }^{1-4}$ In particular, under $\mathrm{cw}$ irradiation fluorescein-doped boric acid glass has been shown to saturate at intensities as low as $15 \mathrm{~mW} / \mathrm{cm}^{2}$, and values for the third-order susceptibility of the order of 1 esu have been reported. ${ }^{1}$ Other organic doped solid materials whose nonlinear-optical properties have been similarly characterized are acridine dyes in lead-tin fluorophosphate glass ${ }^{2}$ and eosin and erythrosin ${ }^{3}$ in gelatin films. In these and similar systems, excitation from the singlet ground state $S_{0}$ to the first singlet excited state $S_{1}$ (absorption cross section $\sigma_{0}$ ) is followed by efficient intersystem crossing of most of the excited population to the long-lived lowest triplet state $T_{1}$, thus making $\mathrm{cw}$ saturation easy to attain. Subsequent intersystem crossing from the vibrationally excited states of the $T_{1}$ manifold back to the first singlet excited state gives rise to delayed fluorescence. This in turn leads to a depopulation of the triplet state on a time scale of the order of $0.1-1.0 \mathrm{~s}$. The decay of the triplet-state population has been found to be nonexponential, which can be attributed to site-to-site variations in the local environment of the dye. The response and decay times of nonlinearities induced by this mechanism of saturation are dependent on the intersystem crossing time and the triplet-state lifetime. They are therefore on a millisecond time scale and thus comparatively slow. Previous experiments in fluoresceindoped boric acid glass employed cw lasers in order to saturate the lowest triplet state. In this case the saturation intensity is $I_{\text {sat }}=\hbar \omega / \sigma_{0} \tau_{T}$, where $\tau_{T}$ is the triplet-state lifetime and $\hbar \omega$ is the photon energy. With the use of short pulses at low repetition rates $\left(<0.2 \mathrm{~s}^{-1}\right)$, buildup of a triplet-state population should be avoided and saturation of the first singlet excited level will be made possible if the pulse width $\Delta t$ is longer than the $S_{1}$ lifetime $\tau$. Saturation intensities are then given by $I_{\text {sat }}=\hbar \omega / \sigma_{0} \tau$. If $\Delta t<\tau$, however, the system simply integrates over the number of photons absorbed and $I_{s}=\hbar \omega / \sigma_{0} \Delta t$ holds. ${ }^{5} \quad$ To our knowledge, the nonlinear-optical behavior of these materials with the use of short (i.e., picosecond) pulses has not been studied yet. Here we report the results of investigations of nonlinear-optical processes in fluorescein-doped boric acid glass that occur on a much shorter time scale than that previously measured. The third-order nonlinear optical susceptibility $\chi^{(3)}$ was measured by degenerate fourwave mixing (DFWM) and nonlinear saturation studies and is of the order of $10^{-7}$ esu with 200 -ps pulses.

\section{SAMPLE AND EXPERIMENTAL DETAILS}

The samples studied were films of fluorescein-doped boric acid glass $\left(8 \times 10^{-3} \mathrm{M}\right.$ concentration) sandwiched between two microscope slides. The preparation method and the absorption and fluorescence spectra are identical to those reported by Kramer et al. in Ref. 1. Pulses of $\sim 200$-ps duration from a modified PRA nitrogen-pumped dye laser (LN1000/LN107) were used throughout. The laser dye was Coumarin 120, which has a peak output at $\sim 444 \mathrm{~nm}$, the wavelength used if not otherwise stated. At this wavelength the ground-state and excited-state absorption cross sections were $\sigma_{0}=1.2 \times 10^{-15} \mathrm{~cm}^{2}$ and $\sigma_{\text {ex }}=0.6 \times$ $10^{-15} \mathrm{~cm}^{2}$, respectively, as determined from absorption spectra and nonlinear saturation (see Section 3). We performed nonlinear saturation experiments by placing the sample in the focus of a $30-\mathrm{cm}$ lens, varying the incident intensity with a variable neutral-density filter, and measuring the transmission with silicon photodiodes. DFWM has been widely used as a means of investigating nonlinear-optical mechanisms in many types of materials. In addition to providing the magnitude of nonlinear-optical coefficients, it has been associated with a range of applications including optical signal processing, real-time holography, information storage, and image correction. Extensive reviews cover these and other aspects of DWFM. ${ }^{6-8}$ Generally, two coherent laser beams are made to interfere in the sample, thus creating a laser-induced grating. This grating is subsequently read out with a further beam, and the diffracted signal is detected. There exist many variations of this standard DFWM geometry, some of which were used in our experiments on the fluorescein-doped films and are described in detail in Section 3. 


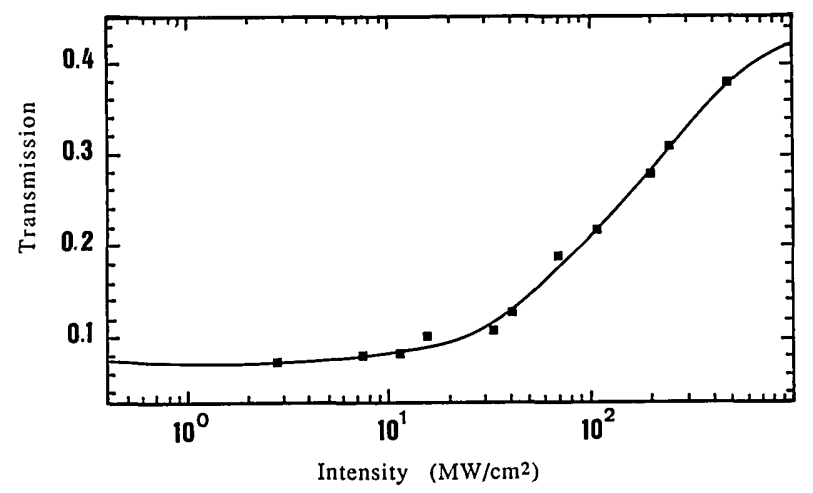

Fig. 1. Intensity-dependent transmission of the fluorescinedoped boric acid glass. The solid curve is a computer fit of the experimental data using a rate-equation model as described in Ref. 5.

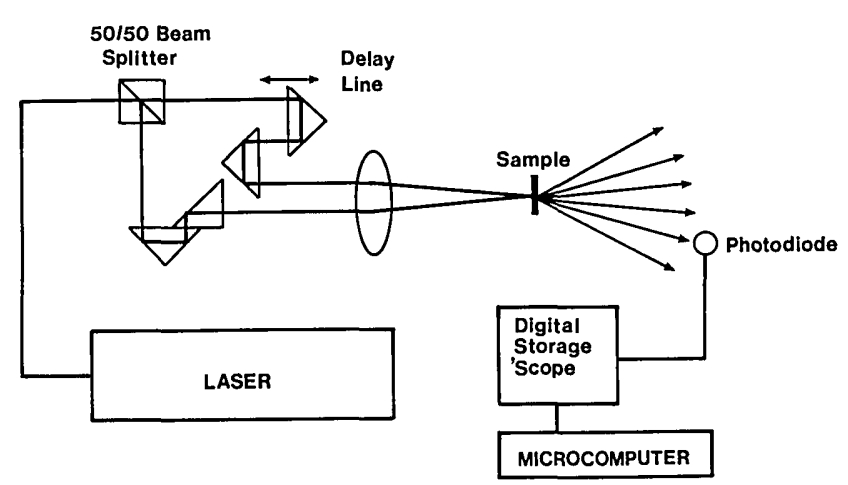

Fig. 2. Experimental setup for forward DFWM.

\section{NONLINEAR-OPTICAL BEHAVIOR}

The sample transmission as a function of incident laser intensity is shown in Fig. 1. The saturation intensity (i.e., that intensity at which the absorption coefficient drops to one half of its unsaturated value) is $\sim 5 \times 10^{7} \mathrm{~W} / \mathrm{cm}^{2}$. Saturation curves were taken at three laser repetition periods (ranging from $250 \mathrm{~ms}$ to $3.6 \mathrm{~s}$ ) that span the $T_{1}$ lifetime in order to establish whether intersystem crossing and delayed fluorescence significantly affected the sample transmission at a particular intensity. Within experimental error no difference between the curves was apparent; thus it could safely be assumed that the transmission was independent of the laser repetition period over this range. Furthermore, this shows that the saturation originates in the singlet system and is rather insensitive to these transitions. The sample is characterized by a saturated transmission saturation well below unity, indicating that excited singlet-singlet transitions are possible. From simple considerations of a saturated groundstate absorption and a nonsaturable excited state, we can estimate the excited-state absorption cross section $\sigma_{\text {ex }}$ from the transmission $T_{\text {ex }}$ achieved as ${ }^{9}$

$$
\sigma_{\mathrm{ex}}=\sigma_{0} \ln \left(T_{\mathrm{ex}} / T_{0}\right),
$$

where $T_{0}$ is the unsaturated transmission. This leads to the values of $\sigma_{\mathrm{ex}}$ quoted in Section 2. As these become significant only at intensities well above $I_{\text {sat }}$, they are of no major concern for DFWM measurements described below. A value for the singlet lifetime that is larger than the pulse width ( $>0.2 \mathrm{~ns})$ can be estimated from the saturation intensity.

The technique of forward DFWM ${ }^{10}$ may be used as a quick and simple means of measuring $\chi^{(3)}$. It is best suited to thin, highly absorbing samples and was therefore employed here to carry out magnitude measurements. The experimental setup is illustrated in Fig. 2. The two incident pump pulses of intensities denoted by $I_{1}$ and $I_{2}$ are focused by a $30-\mathrm{cm}$ lens to a diameter of $\sim 400 \mu \mathrm{m}$. They overlap in the sample to create an intensity modulation $\left(I_{1}-I_{2}\right) /\left(I_{1}+I_{2}\right)$. A variable optical delay line serves to achieve temporal overlap to within the coherence time of the dye laser pulse $(\sim 3.0 \mathrm{ps})$. If the sample exhibits a significant intensity-dependent refractive index, self-diffraction of each pulse from an induced refractive index grating occurs, yielding one or more signal pulses to either side of the transmitted (or zero-order) pulses. The exact position of these signals is determined by momentum conservation. The angle between the incident pulses is kept small in order that the thin-grating condition be satisfied. $^{6}$ When this is the case, the Bragg condition is relaxed and diffraction efficiency becomes independent of incident angles. Of interest to us is the first-order signal, as this can be used to give an estimate of $\chi^{(3)}$. Higherorder signals are due to a combination of higher-order susceptibilities and higher diffracted orders. $\chi^{(3)}$ can be expressed in terms of diffraction efficiency $\eta$ as

$$
\left|\chi^{(3)}\right|=\frac{4 \epsilon_{0} c \alpha n^{2} \lambda \eta^{1 / 2}}{3 \pi T^{1 / 2}(1-T) I_{0}},
$$

where $T$ is the film transmission at the total incident intensity $I_{0}$. A derivation of Eq. (2) is given in Appendix A.

The dependence of the diffraction efficiency on input intensity was measured and is shown in Fig. 3. For a third-order nonlinearity a square dependence would be expected; however, from inspection of the intensitydependent transmission of the sample (shown in Fig. 1), one can expect that the increase in transmission at higher intensities leads to reduced coupling of the input pulses and a consequently lower diffraction efficiency. From this measurement and using Eq. (2), one can estimate a value for $\chi^{(3)}$ of $9 \times 10^{-8}$ esu at $444 \mathrm{~nm}$. The wavelength dependence of $\chi^{(3)}$, when it is normalized to the laser output, and the absorption coefficient of the sample follow the

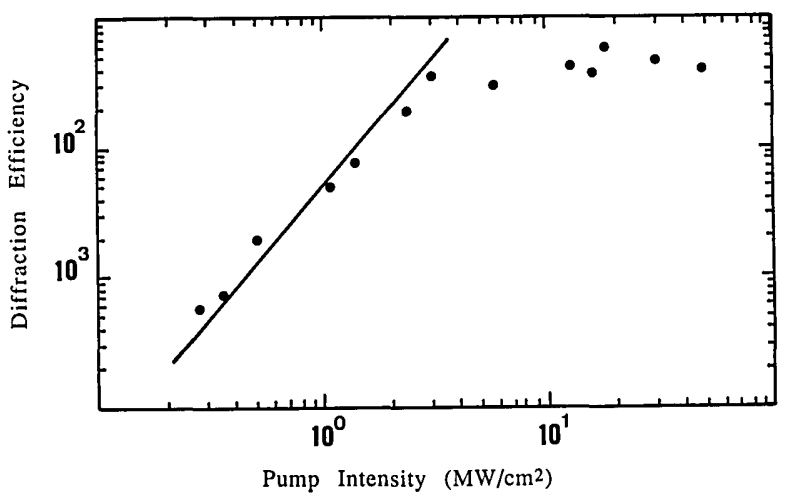

Fig. 3. Intensity dependence of the diffraction efficiency in forward four-wave mixing showing saturation at high intensities. The solid portion depicts a line with slope 2 as predicted by Eq. (2). 


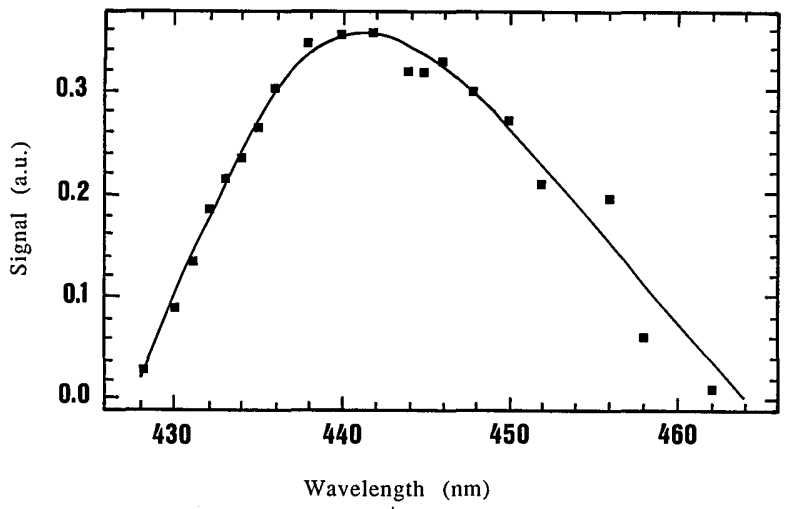

Fig. 4. Wavelength dependence of the nonlinear susceptibility $\chi^{(3)}$. The solid line represents a guide to the eye.

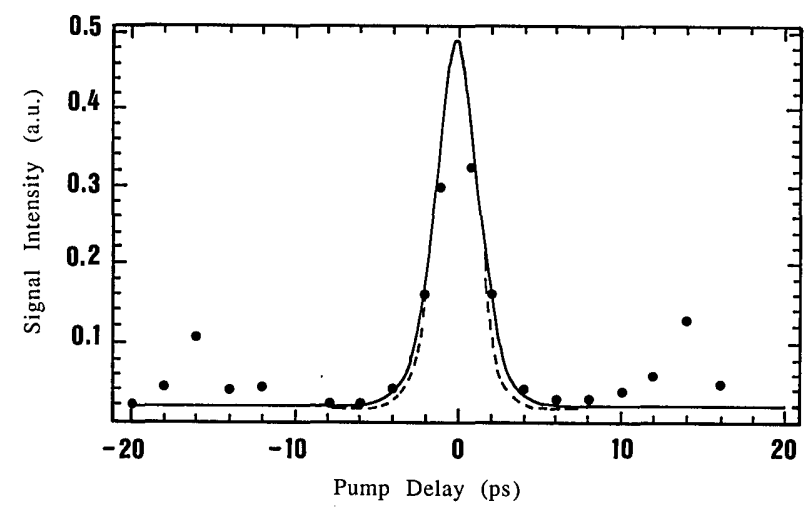

Fig. 5. Diffracted signal intensity as a function of the writingbeam delay in forward four-wave mixing. The solid curve depicts a fit to a squared hyperbolic secant temporal pulse profile, and the dashed curve represents a fit to a Gaussian pulse shape.

absorption (see Fig. 4). At long wavelengths the diffraction efficiency decreases owing to loss of writing beam coupling, and at short wavelengths the absorption is too large. In the forward DFWM technique, diffraction from the induced grating and creation of the grating are taking place simultaneously; therefore only a limited amount of temporal information can be gained by a delay of incidence of one of the pulses. In Fig. 5 we show a plot of diffracted signal intensity as a function of delay of incidence of one of the pulses. The width of the peak is $2.9 \mathrm{ps}$, which corresponds to the temporal coherence of the dye laser. Fits to both a Gaussian and a squared hyperbolic secant pulse shape are given. These fits idicate that the grating lifetime $\tau_{g}$ is longer than the temporal coherence $\tau_{c}$ of the laser. ${ }^{11}$ If, on the other hand, $\tau_{g}<\tau_{c}$, then similar measurements would yield the intensity autocorrelation of the incident laser pulse. (The peaks to either side of the central peak are due to a coherence substructure in the laser output.) Hence a preferable means of probing the lifetime of the induced nonlinearity is to use a third independent readout beam. For this procedure a probe beam $I_{3}$ was split off from the main beam, passed through a variable optical delay line, and directed through the lens traveling parallel to $I_{1}$ and $I_{2}$, but in such a way that the three beams were positioned at three corners of a square on the lens surface. In this manner one could switch from the two-beam to the three-beam arrangement with minimum disturbance. As momentum conservation would predict, the diffracted signal $I_{3 d}$ due to this probe pulse is observed at the fourth corner of the square. The presence of the self-diffracted signal due to the two pumps is useful, since their alignment can be optimized in the presence of the probe. Also, $I_{3 d}$ is much more difficult to observe simply because $I_{3}$ is much weaker than $I_{1}$ or $I_{2}$. By monitoring the intensity of $I_{3 d}$ as a function of incidence of $I_{3}$ with respect to $I_{1}$ and $I_{2}$ at the sample, one can obtain the lifetime of the induced grating. This arrangement is commonly termed folded boxcars geometry. ${ }^{12}$ As Fig. 6 shows, the transient grating is found to decay with a time constant of $1.2 \pm 0.3 \mathrm{~ns}$. The $S_{1}$ lifetime is therefore twice this value: $\tau=2.4 \pm 0.6 \mathrm{~ns}$.

\section{FLUORESCENCE MEASUREMENTS AND LASER REPETITION RATE DEPENDENCE}

With the exception of the transmission saturation mentioned at the beginning of Section 3 , all the measurements described above have been carried out at a repetition period of $800 \mathrm{~ms}$. Figure 7(a) shows the variation of $\chi^{(3)}$ with laser repetition period. As a comparison, Fig. 7(b) shows the variation of the sample fluorescence (as measured with a photodiode) with laser repetition period. Both signals increase wih the laser repetition period to reach a plateau at periods greater than $1.0 \mathrm{~s}$. This suggests that, at repetition periods less than or close to the $T_{1}$ triplet-state lifetime, a triplet population builds up, reducing the singlet population and thus decreasing the fluorescence. As the repetition period is increased, the triplet population cannot grow, and the $S_{1}$ population relaxes directly to $S_{0}$ by fluorescence. The intersystem crossing time can be estimated from the triplet quantum yield ${ }^{1}$ $(<0.9)$ and the $S_{1}$ lifetime and appears to be slower than the pulse width (i.e., $>0.2 \mathrm{~ns}$ ). As a result, we can confirm that in the four-wave-mixing experiments it is the induced $S_{1}$ population that gives rise to the transient grating and hence to the diffracted signal. Since we were working in the absorption maximum at intensities below saturation, the nature of the grating will be predominantly absorptive and the lifetime of the induced grating corresponds to the lifetime of the singlet state as measured. To check this nanosecond decay, we used a fast silicon photodiode combined with a Tektronix 7834 storage oscilloscope (combined time resolution $5 \mathrm{~ns}$ ) to study the dynamics of fluorescence directly. With all the repetition rates we find a fast $(<5 \mathrm{~ns})$ decay, in agreement with the time-

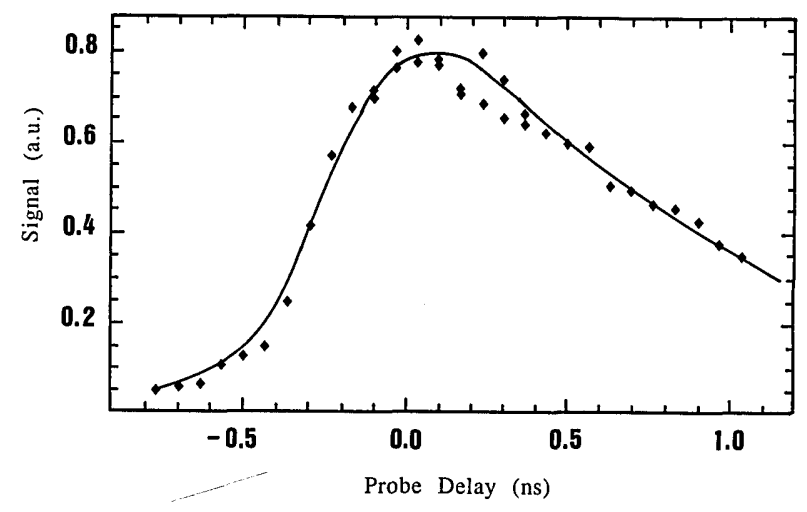

Fig. 6. Temporal decay of the diffracted intensity as measured in the folded boxcars geometry. The solid curve is a computer fit using a laser-pulse duration of 200 ps and a grating decay time of $1.2 \mathrm{~ns}$. 


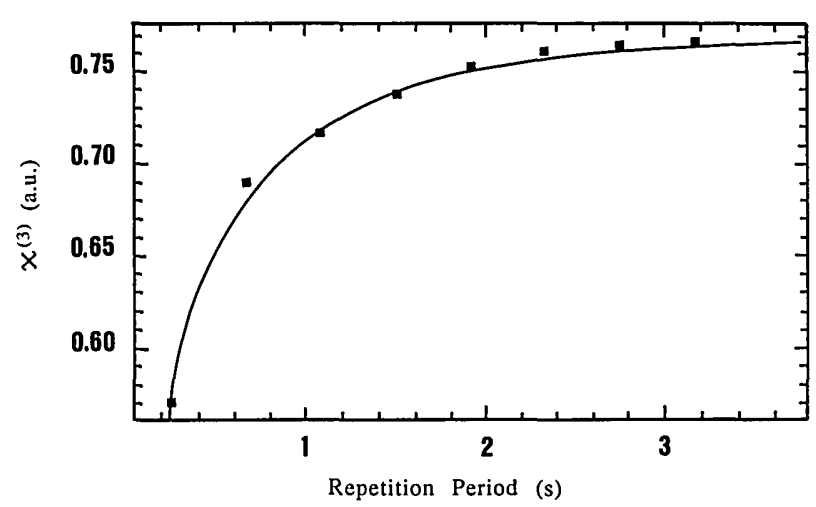

(a)

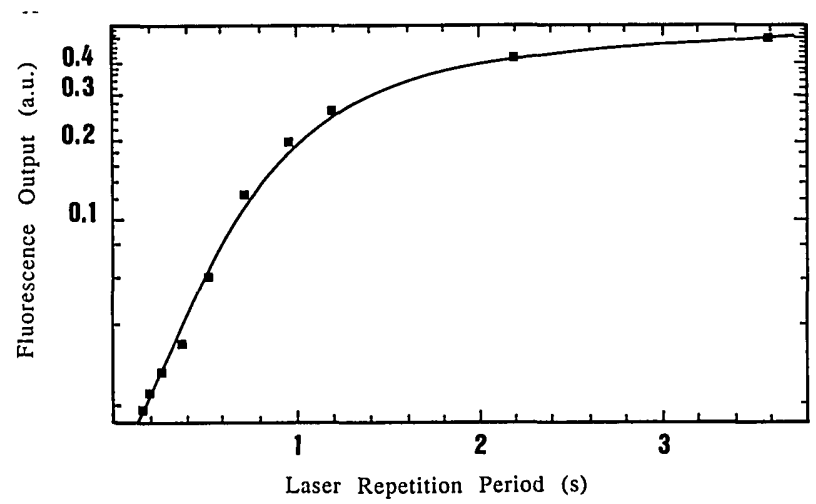

(b)

Fig. 7. (a) Variation of nonlinear susceptibility $\chi^{(3)}$ with laser repetition period. (b) Variation of sample fluorescence with laser repetition period. In both plots the solid curve represents a guide to the eye.

resolved DFWM measurement described in Section 3. For repetition rates slower than $0.8 \mathrm{~s}$, however, a second, slower component with a decay time of $\sim 15 \mathrm{~ns}$ is observed. We cannot attribute this time to the delayed fluorescence, as the buildup of the triplet-state population should increase with increasing repetition period. A possible explanation for this signal is fluorescence of a set of fluorescein molecules that are in a specific site of the glass matrix, giving them a longer decay time owing to local environmental effects. Such effects can indeed be observed in glass matrices. ${ }^{13}$ However, the time needed to populate this state must be slower than $\sim 1 \mathrm{~s}$.

\section{CONCLUSIONS}

We have observed that with short-pulse excitation optical saturation and efficient four-wave mixing can be observed through the singlet state of fluorescein-doped boric acid glass. The triplet-state population reduces the number of molecules in the singlet state and thus reduces the fourwave mixing signal. Nevertheless, we have observed sizable nonlinearities $\left(\sim 10^{-7}\right.$ esu) that make these materials interesting also for high-power, short-pulse applications.

\section{APPENDIX A: DERIVATION OF $\left|\chi^{(3)}\right|$ FROM EXPERIMENTALLY MEASURED PARAMETERS FOR THE FORWARD DEGENERATE FOUR-WAVE MIXING GEOMETRY}

The phenomenon of self-diffraction occurs as a result of the modulation of the optical properties of a material by two light beams owing to nonlinear optical processes and the subsequent diffraction from the periodic modulation. The degree of diffraction is therefore a measure of the susceptibility of the material properties to modulation.

The complex refractive index $\kappa$ of the material can be expressed in terms of the material susceptibility $\chi$ :

$$
\kappa^{2}=1+\chi
$$

The change $\Delta \kappa$ induced in the material owing to a nonlinear interaction with an incident light field is therefore given by

$$
\Delta \kappa=\Delta \chi / 2(1+\chi)^{1 / 2} \text {. }
$$

In the formalism of nonlinear optics the input radiation creates a nonlinear polarization

$$
\begin{aligned}
P_{i} & =\frac{1}{2} \epsilon_{0}\left[\chi_{i j}^{(1)} A_{j}+\frac{3}{4} \chi_{i j k l}^{(3)} A_{j} A_{k} A_{l}+\ldots\right] \\
& =\frac{1}{2} \epsilon_{0}\left[\chi_{i j}^{(1)} A_{j}+\Delta \chi_{i j} A_{j}\right]
\end{aligned}
$$

which acts as a source term and reradiates, in accordance with the phase-matching conditions, to produce diffraction. Here $\chi^{(i)}$ is the $i$ th-order susceptibility and $A_{i}$ represents the $i$ th electric-field amplitude. Therefore

$$
\Delta \chi_{i j}=\frac{3}{4} \chi_{i j k l}^{(3)} A_{k} A_{l}+\ldots,
$$

and, according to Eq. (A2),

$$
\Delta \kappa=3 \chi_{i j k l}^{(3)} A_{j} A_{l} / 8 \kappa .
$$

The diffraction efficiency of a laser-induced grating into the first order is given by ${ }^{6}$

$$
I_{d} / I_{c}=\eta=\left|\pi \Delta \kappa d / \lambda_{c}\right|^{2} .
$$

The equation holds for gratings with sufficiently small $|\Delta \kappa|$ and material absorption. For larger material absorptions, $\eta$ is given by

$$
\eta=T I_{d} / I_{c}
$$

where $T$ is the sample transmission.

Hence, for the case in which third-order terms dominate, an expression relating the third-order nonlinear susceptibility to the diffraction efficiency of a laser-induced grating may be derived from Eqs. (A5) and (A7):

$$
\left|\chi^{(3)}\right|=\frac{8 \kappa \lambda \eta^{1 / 2}}{3 \pi d A_{k} A_{l}}
$$

If we convert from field amplitudes to intensity $I$ according to the relationship

$$
A_{k} A_{l}=2 I / \epsilon_{0} c \kappa,
$$

Eq. (A8) becomes

$$
\left|\chi^{(3)}\right|=\frac{4 \epsilon_{0} c \kappa^{2} \lambda \eta^{1 / 2}}{3 \pi d I} .
$$

In absorbing media, for small or intermediate absorption coefficient $\alpha, \alpha^{2} \lambda^{3}$ goes to zero and the expression

$$
\left|\chi^{(3)}\right|=\frac{4 \epsilon_{0} c n^{2} \lambda \alpha \eta^{1 / 2}}{3 \pi I T^{1 / 2}(1-T)}
$$


holds. With the simple two-beam diffraction method, therefore, measurement of the diffracted intensity into the first order can provide a measurement of the thirdorder nonlinear susceptibility.

\section{ACKNOWLEDGMENTS}

The idea for this paper originated during a Window-onScience visit of one of the authors (W.B.) to the U.S. Air Force Weapons Laboratory, Kirtland Air Force Base, Albuquerque, New Mexico, sponsored by the European office for Aerospace Research and Development, London. Discussions with D. Martinez and L. P. Schelonka there and the donation of the samples by them are gratefully acknowledged.

*Present address, Central Research Laboratories, Ciba Geigy AG, Basel, Switzerland.

\section{REFERENCES}

1. M. A. Kramer, W. R. Tompkin, and R. W. Boyd, Phys. Rev. A 34, 2026 (1986).
2. W. R. Tompkin, R. W. Boyd, D. W. Hall, and P. A. Tick, J. Opt. Soc. Am. B 4, 1030 (1987).

3. Y. Silberberg and I. Bar-Joseph, Opt. Commun. 39, 265 (1981).

4. S. A. Boothroyd, J. Chrostowski, and M. S. O'Sullivan, J. Opt. Soc. Am. B 6, 766 (1989).

5. A. Penzkofer and W. Blau, Opt. Quantum Electron. 15, 325 (1983).

6. H. E. Eichler, P. Günter, and D. W. Pohl, Laser-Induced Dynamic Gratings (Springer-Verlag, Berlin, 1986).

7. R. A. Fischer, ed., Optical Phase Conjugation (Academic, New York, 1983).

8. C. Flytzanis and J. L. Oudar, eds., Nonlinear Optics: Materials and Devices, Springer Proceedings in Physics 7 (Springer-Verlag, Berlin, 1986).

9. W. Blau, H. Byrne, and J. M. Kelly, Opt. Commun. 56, 25 (1985).

10. P. Horan, W. Blau, H. Byrne, and P. Berglund, Appl. Opt. 29, 31 (1990).

11. M. A. Vasil'eva, J. Vischakas, V. Kabelka, and A. V. Masalov, Opt. Commun. 53, 412 (1985).

12. See, e.g., G. M. Carter, J. Opt. Soc. Am. B 4, 1018 (1987).

13. M. Frakowiak and H. Walerys, Acta Phys. Polon. 18, 93 (1959). 